PROCEEDINGS OF THE

AMERICAN MATHEMATICAL SOCIETY

Volume 137, Number 12, December 2009, Pages 4117-4127

S 0002-9939(09)09973-0

Article electronically published on June 19, 2009

\title{
ATTRACTIVITY FOR TWO-DIMENSIONAL LINEAR SYSTEMS WHOSE ANTI-DIAGONAL COEFFICIENTS ARE PERIODIC
}

\author{
JITSURO SUGIE AND AYANO ENDO
}

(Communicated by Yingfei Yi)

\begin{abstract}
This paper deals with the linear system $\mathbf{x}^{\prime}=A(t) \mathbf{x}$ with $A(t)$ being a $2 \times 2$ matrix. The anti-diagonal components of $A(t)$ are assumed to be periodic, but the diagonal components are not necessarily periodic. Our concern is to establish sufficient conditions for the zero solution to be attractive. Floquet theory is of no use in solving our problem, because not all components are periodic. Another approach is adopted. Some simple examples are included to illustrate the main result.
\end{abstract}

\section{INTRODUCTION}

We consider the linear system

$$
\mathbf{x}^{\prime}=A(t) \mathbf{x}=\left(\begin{array}{cc}
-r(t) & p(t) \\
-p(t) & -q(t)
\end{array}\right) \mathbf{x},
$$

where the prime denotes $d / d t$; the coefficients $p(t), q(t)$ and $r(t)$ are continuous for $t \geq 0$, and $p(t)$ is a periodic function with period $\omega>0$. The coefficients $q(t)$ and $r(t)$ are not always assumed to be periodic. Since system (11) has such a simple form, it has broad applications to science and engineering.

It is well-known that the zero solution of (1) is asymptotically stable if it is attractive; that is, every solution $\mathbf{x}(t)$ of (1) tends to $\mathbf{0} \in \mathbb{R}^{2}$ as $t \rightarrow \infty$. The purpose of this paper is to give sufficient conditions on $p(t), q(t)$ and $r(t)$ which guarantee the attractivity of the zero solution of (1).

Floquet's theorem is available for the special case where $q(t)$ and $r(t)$ are also periodic functions with period $\omega$. Let $\Phi(t)$ be the fundamental matrix of (1) with $\Phi(0)=E$, the $2 \times 2$ identity matrix. Then $\Phi(\omega)$ is called the monodromy matrix of (1). Let $\mu_{1}$ and $\mu_{2}$ be the eigenvalues of the monodromy matrix $\Phi(\omega)$. The eigenvalues $\mu_{1}$ and $\mu_{2}$ are often called the Floquet multipliers of (1). By Abel's formula,

$$
\operatorname{det} \Phi(\omega)=\operatorname{det} \Phi(0) \exp \left(-\int_{0}^{\omega}(q(s)+r(s)) d s\right)=\exp \left(-\int_{0}^{\omega}(q(s)+r(s)) d s\right) .
$$

Received by the editors March 17, 2009.

2000 Mathematics Subject Classification. Primary 34D05, 34D20; Secondary 37B25, 37C75.

Key words and phrases. Attractivity, linear systems, weakly integrally positive, Floquet theory.

The first author was supported in part by a Grant-in-Aid for Scientific Research, No. 19540182, from the Japan Society for the Promotion of Science.

(C)2009 American Mathematical Society Reverts to public domain 28 years from publication 
Thus, the Floquet multipliers $\mu_{1}$ and $\mu_{2}$ are the roots of the equation

$$
\mu^{2}-\operatorname{tr} \Phi(\omega) \mu+\exp \left(-\int_{0}^{\omega}(q(s)+r(s)) d s\right)=0 .
$$

It follows from Floquet's theorem that the zero solution of (11) with periodic coefficients $q(t)$ and $r(t)$ is attractive if and only if the Floquet multipliers $\mu_{1}$ and $\mu_{2}$ have magnitude strictly less than 1 . Hence, in this special case, necessary and sufficient conditions for the zero solution of (1) to be attractive are that

$$
|\operatorname{tr} \Phi(\omega)|<1+\exp \left(-\int_{0}^{\omega}(q(s)+r(s)) d s\right)
$$

and

$$
\exp \left(-\int_{0}^{\omega}(q(s)+r(s)) d s\right)<1 .
$$

For example, we can find Floquet's theorem in the books [2, 3, 5, 8, 16.

Although the above conditions are necessary and sufficient for the zero solution of (11) to be attractive, it is difficult to estimate the absolute value of the trace of $\Phi(\omega)$, because it is impossible to find a fundamental matrix of (1) in general. Of course, Floquet's theorem is useless when $q(t)$ or $r(t)$ is not periodic. Then, without knowledge of a fundamental matrix of (11), can we decide whether the zero solution is attractive? What kind of condition on $A(t)$ will guarantee the attractivity of the zero solution of (1)?

We give an answer to our question in Sections 2 and 3. In Section 2, we state the main result and present some preparatory lemmas. In Section 3, we give the proof of the main result. To illustrate our main result, we take some concrete examples and exhibit positive orbits of (11) in Section 4. In addition, we mention the approach via Floquet theory.

\section{Some Lemmas}

Let

$$
R(t)=\int_{0}^{t} r(s) d s \quad \text { and } \quad \psi(t)=2(q(t)-r(t))
$$

for $t \geq 0$. For the sake of convenience, we write

$$
\psi_{+}(t)=\max \{0, \psi(t)\} \quad \text { and } \quad \psi_{-}(t)=\max \{0,-\psi(t)\} .
$$

Note that $\psi(t)=\psi_{+}(t)-\psi_{-}(t)$ and $|\psi(t)|=\psi_{+}(t)+\psi_{-}(t)$. If $r(t) \equiv 0$ and $p(t) \equiv k>0$, then $\psi(t)=2 q(t)$ and system (1) is equivalent to the damped linear oscillator of one degree of freedom,

$$
x^{\prime \prime}+q(t) x^{\prime}+k^{2} x=0 .
$$

It is clear that the equilibrium $\left(x, x^{\prime}\right)=(0,0)$ of (2) corresponds to the zero solution of (11). It is well-known that the divergence of an indefinite integral of $q(t)$ is not sufficient to guarantee that the equilibrium of (2) will be attractive. For this reason, it is natural to make a stronger assumption on $\psi(t)$.

We introduce an important concept here. A nonnegative function $\phi(t)$ is said to be weakly integrally positive if

$$
\int_{I} \phi(t) d t=\infty
$$


for every set $I=\bigcup_{n=1}^{\infty}\left[\tau_{n}, \sigma_{n}\right]$ such that $\tau_{n}+\delta<\sigma_{n}<\tau_{n+1}<\sigma_{n}+\Delta$ for some $\delta>0$ and $\Delta>0$. For example, $1 /(1+t)$ and $\sin ^{2} t /(1+t)$ are weakly integrally positive functions (see [6, 7, 13, 14, 15]).

Our main result is as follows:

Theorem 1. Suppose that $q(t)$ and $R(t)$ are bounded for $t \geq 0$. Suppose also that

(i) $\psi_{+}(t)$ is weakly integrally positive;

(ii) $\int_{0}^{\infty} \psi_{-}(t) d t<\infty$.

Then the zero solution of (1) is attractive.

Before proving our result, we present some lemmas.

Lemma 2. Suppose that assumption (ii) in Theorem 1 holds. Let $v(t)$ be nonnegative and continuously differentiable on $\left[t_{0}, \infty\right)$ for some $t_{0}>0$. If

$$
v^{\prime}(t) \leq \psi_{-}(t) v(t) \quad \text { for } t \geq t_{0}
$$

then $v^{\prime}(t)$ is absolutely integrable, and therefore $v(t)$ has a nonnegative limiting value.

Proof. By (3), we have

$$
\left(v(t) \exp \left(-\int_{t_{0}}^{t} \psi_{-}(s) d s\right)\right)^{\prime}=\left(v^{\prime}(t)-\psi_{-}(t) v(t)\right) \exp \left(-\int_{t_{0}}^{t} \psi_{-}(s) d s\right) \leq 0
$$

for $t \geq t_{0}$. Integrating this inequality from $t_{0}$ to $t$, we obtain

$$
v(t) \leq v\left(t_{0}\right) \exp \left(\int_{t_{0}}^{t} \psi_{-}(s) d s\right) \text { for } t \geq t_{0} .
$$

Hence, using (3) again, we get

$$
v^{\prime}(t) \leq v\left(t_{0}\right) \exp \left(\int_{t_{0}}^{t} \psi_{-}(s) d s\right) \psi_{-}(t) \text { for } t \geq t_{0} .
$$

It follows from assumption (ii) that

$$
v^{\prime}(t) \leq v\left(t_{0}\right) \exp \left(\int_{t_{0}}^{\infty} \psi_{-}(s) d s\right) \psi_{-}(t) \quad \text { for } t \geq t_{0}
$$

Since the right-hand side of the above inequality is positive for $t \geq t_{0}$, we see that

$$
\left(v^{\prime}\right)_{+}(t) \leq v\left(t_{0}\right) \exp \left(\int_{t_{0}}^{\infty} \psi_{-}(s) d s\right) \psi_{-}(t)
$$

Consequently,

$$
\int_{t_{0}}^{\infty}\left(v^{\prime}\right)_{+}(s) d s \leq v\left(t_{0}\right) \exp \left(\int_{t_{0}}^{\infty} \psi_{-}(s) d s\right) \int_{t_{0}}^{\infty} \psi_{-}(s) d s<\infty .
$$

On the other hand, since $v(t) \geq 0$ for $t \geq t_{0}$, we get

$$
\int_{t_{0}}^{\infty}\left(v^{\prime}\right)_{-}(s) d s=\int_{t_{0}}^{\infty}\left(v^{\prime}\right)_{+}(s) d s-\int_{t_{0}}^{\infty} v^{\prime}(s) d s \leq \int_{t_{0}}^{\infty}\left(v^{\prime}\right)_{+}(s) d s+v\left(t_{0}\right)<\infty .
$$


Hence, we obtain

$$
\int_{t_{0}}^{\infty}\left|v^{\prime}(s)\right| d s=\int_{t_{0}}^{\infty}\left(\left(v^{\prime}\right)_{+}(s)+\left(v^{\prime}\right)_{-}(s)\right) d s<\infty .
$$

Since $v(t)$ is nonnegative for $t \geq t_{0}$ and $v^{\prime}(t)$ is absolutely integrable, it turns out that $v(t)$ has a limiting value $v_{0} \geq 0$. This completes the proof of Lemma 2 .

Using a classical Lyapunov's direct method, we can prove that all solutions of (11) are uniformly bounded; that is, for any $\alpha>0$, there exists a $\beta(\alpha)>0$ such that $t_{0} \geq 0$ and $\left\|\mathbf{x}_{0}\right\|<\alpha$ imply $\left\|\mathbf{x}\left(t ; t_{0}, \mathbf{x}_{0}\right)\right\|<\beta$ for all $t \geq t_{0}$. For details about the direct method of Lyapunov, see the books [1, 2, 4, 5, 9, 10, 11, 12, 17, 18, 19, for example.

Lemma 3. Suppose that $R(t)$ is bounded for $t \geq 0$. If assumption (ii) in Theorem 1 holds, then all solutions of (11) are uniformly bounded.

Proof. Let $\mathbf{x}=(x, y)$ and define two Lyapunov functions

and

$$
V(t, \mathbf{x})=\frac{1}{2} e^{2 R(t)}\left(x^{2}+y^{2}\right)
$$

$$
U(t, \mathbf{x})=V(t, \mathbf{x}) \exp \left(-\int_{0}^{t} \psi_{-}(s) d s\right)
$$

on $[0, \infty) \times \mathbb{R}^{2}$. From the boundedness of $R(t)$, we can choose an $L>0$ such that $|R(t)|<L$ for $t \geq 0$. Let

$$
M=\int_{0}^{\infty} \psi_{-}(s) d s
$$

(because of assumption (ii), such an $M$ exists). Then, we have

$$
\frac{1}{2} e^{-(2 L+M)}\left(x^{2}+y^{2}\right) \leq V(t, \mathbf{x}) e^{-M} \leq U(t, \mathbf{x}) \leq V(t, \mathbf{x}) \leq \frac{1}{2} e^{2 L}\left(x^{2}+y^{2}\right) .
$$

Thus, $U(t, \mathbf{x})$ tends to $\infty$ as $\|\mathbf{x}\| \rightarrow \infty$ uniformly for $t \geq 0$ (i.e. it is radially unbounded), and it is decrescent. Differentiate $V(t, \mathbf{x})$ along any solution of (1) to obtain

$$
\dot{V}_{\text {(1) }}(t, \mathbf{x})=-\left((q(t)-r(t)) e^{2 R(t)} y^{2} \leq \psi_{-}(t) V(t, \mathbf{x})\right.
$$

on $[0, \infty) \times \mathbb{R}^{2}$. Hence, we have

$$
\dot{U}_{\mathbb{1}}(t, \mathbf{x})=\left\{\dot{V}_{\mathbb{1}}(t, \mathbf{x})-\psi_{-}(t) V(t, \mathbf{x})\right\} \exp \left(-\int_{0}^{t} \psi_{-}(s) d s\right) \leq 0 .
$$

We therefore conclude that all solutions of (1) are uniformly bounded by using a Lyapunov-type theorem due to Yoshizawa [17, 18, 19].

Remark 1. Using the same Lyapunov function $U(t, \mathbf{x})$, we can prove that the zero solution of (11) is uniformly stable.

Recall that $p(t)$ is a periodic function with period $\omega>0$. Let

$$
\bar{p}=\max _{t \in[0, \omega]} p(t) \quad \text { and } \quad \underline{p}=\min _{t \in[0, \omega]} p(t) .
$$

Taking $\bar{p} \geq \underline{p}$ into account, we see that if $\bar{p}+\underline{p} \geq 0$, then $\bar{p}>0$; if $\bar{p}+\underline{p}<0$, then $\underline{p}<0$. Since $p(t)$ is continuous for $t \geq 0$, we see that $p(t)$ has the following property (we omit the proof). 
Lemma 4. Suppose that $p(t)$ is a nontrivial periodic function with period $\omega>0$. If $\bar{p}+\underline{p} \geq 0$, then there exist numbers $a$ and $b$ with $0 \leq a<b \leq \omega$ such that

$$
p(t) \geq \frac{1}{2} \bar{p}>0 \quad \text { for } a \leq t \leq b .
$$

If $\bar{p}+\underline{p}<0$, then there exist numbers $a$ and $b$ with $0 \leq a<b \leq \omega$ such that

$$
p(t) \leq \frac{1}{2} \underline{p}<0 \quad \text { for } a \leq t \leq b .
$$

Remark 2. Let $m$ be any integer. Since $p(t)$ is a periodic function with period $\omega>0$, it turns out that if $\bar{p}+\underline{p} \geq 0$, then

$$
p(t) \geq \frac{1}{2} \bar{p}>0 \text { for } a+m \omega \leq t \leq b+m \omega ;
$$

if $\bar{p}+\underline{p}<0$, then

$$
p(t) \leq \frac{1}{2} \bar{p}<0 \text { for } a+m \omega \leq t \leq b+m \omega .
$$

\section{Proof of the main Result}

We are now ready to prove Theorem 1.

Proof of Theorem 1. Let $\mathbf{x}\left(t ; t_{0}, \mathbf{x}_{0}\right)$ be a solution of (1) passing through $\left(t_{0}, \mathbf{x}_{0}\right) \in$ $[0, \infty) \times \mathbb{R}^{2}$. It follows from Lemma 3 that for any $\alpha>0$, there exists a $\beta(\alpha)>0$ such that $t_{0} \geq 0$ and $\left\|\mathbf{x}_{0}\right\|<\alpha$ imply that

$$
\left\|\mathbf{x}\left(t ; t_{0}, \mathbf{x}_{0}\right)\right\|<\beta \text { for } t \geq t_{0} .
$$

For the sake of brevity, we write $(x(t), y(t))=\mathbf{x}\left(t ; t_{0}, \mathbf{x}_{0}\right)$ and

$$
v(t)=V(t, x(t), y(t)) \text {. }
$$

Then, we have

$$
v(t)=\frac{1}{2} e^{2 R(t)}\left(x^{2}(t)+y^{2}(t)\right)
$$

and

$$
v^{\prime}(t)=-\left((q(t)-r(t)) e^{2 R(t)} y^{2} \leq \psi_{-}(t) v(t)\right.
$$

for $t \geq t_{0}$ (see the calculation of $\dot{V}_{\text {(1) }}(t, \mathbf{x})$ in the proof of Lemma 2). Hence, from Lemma 2 , we see that $v(t)$ has a limiting value $v_{0} \geq 0$. If $v_{0}=0$, then by (15) the solution $(x(t), y(t))$ tends to $\mathbf{0}$ as $t \rightarrow \infty$. This completes the proof. Thus, we need consider only the case in which $v_{0}>0$. We will show that this case does not occur.

Because of (4), we see that $|y(t)|$ is bounded for $t \geq t_{0}$. Hence, $|y(t)|$ has an inferior limit and a superior limit. First, we will show that the inferior limit of $|y(t)|$ is zero, and we will then show that the superior limit of $|y(t)|$ is also zero.

Suppose that $\liminf _{t \rightarrow \infty}|y(t)|>0$. Then, there exist a $\gamma>0$ and a $T_{1} \geq t_{0}$ such that $y(t)>\gamma$ for $t \geq T_{1}$. It follows from (6) and Lemma 2 that

$$
\begin{aligned}
\infty>\int_{t_{0}}^{\infty}\left|v^{\prime}(s)\right| d s & =\frac{1}{2} \int_{t_{0}}^{\infty}|\psi(s)| e^{2 R(s)} y^{2}(s) d s \\
& \geq \frac{1}{2} \gamma^{2} \int_{T_{1}}^{\infty} \psi_{+}(s) e^{2 R(s)} d s \geq \frac{1}{2} \gamma^{2} e^{-2 L} \int_{T_{1}}^{\infty} \psi_{+}(s) d s,
\end{aligned}
$$

where $L$ is the number given in the proof of Lemma 3. This contradicts assumption (i). Thus, we see that $\liminf _{t \rightarrow \infty}|y(t)|=0$. 
Suppose that $\lim \sup _{t \rightarrow \infty}|y(t)|>0$. Let $\nu=\lim \sup _{t \rightarrow \infty}|y(t)|$. Since $q(t)$ is bounded, we can find a $\bar{q}>0$ such that

$$
|q(t)| \leq \bar{q} \text { for } t \geq 0 .
$$

Since $v(t)$ tends to a positive value $v_{0}$ as $t \rightarrow \infty$, there exists a $T_{2} \geq t_{0}$ such that

$$
0<\frac{1}{2} v_{0}<v(t)<\frac{3}{2} v_{0} \quad \text { for } t \geq T_{2} .
$$

Let $\varepsilon$ be so small that

$$
0<\varepsilon<\min \left\{\frac{1}{2} \nu, \sqrt{\frac{\bar{p} e^{-2 L} v_{0}}{4(\bar{q}+2 /(b-a))^{2}+\bar{p}^{2}}}, \sqrt{\frac{\underline{p} e^{-2 L} v_{0}}{4(\bar{q}+2 /(b-a))^{2}+\underline{p}^{2}}}\right\},
$$

where $a$ and $b$ are the numbers given in Lemma 4. Then, since $\lim \inf _{t \rightarrow \infty}|y(t)|=0$, we can select two intervals $\left[\tau_{n}, \sigma_{n}\right]$ and $\left[t_{n}, s_{n}\right]$ with $\left[t_{n}, s_{n}\right] \subset\left[\tau_{n}, \sigma_{n}\right], T_{2}<\tau_{n}$ and $\tau_{n} \rightarrow \infty$ as $n \rightarrow \infty$ such that $\left|y\left(\tau_{n}\right)\right|=\left|y\left(\sigma_{n}\right)\right|=\varepsilon,\left|y\left(t_{n}\right)\right|=\nu / 2,\left|y\left(s_{n}\right)\right|=3 \nu / 4$ and

$$
\begin{gathered}
|y(t)| \geq \varepsilon \text { for } \tau_{n}<t<\sigma_{n}, \\
0 \leq|y(t)| \leq \varepsilon \text { for } \sigma_{n}<t<\tau_{n+1}, \\
\frac{1}{2} \nu<|y(t)|<\frac{3}{4} \nu \text { for } t_{n}<t<s_{n} .
\end{gathered}
$$

By (5), (8) and (11), we have

$$
|x(t)|=\sqrt{2 e^{-2 R(t)} v(t)-y^{2}(t)} \geq \sqrt{e^{-2 L} v_{0}-\varepsilon^{2}}
$$

for $\sigma_{n} \leq t \leq \tau_{n+1}$.

Claim. The sequences $\left\{\tau_{n}\right\}$ and $\left\{\sigma_{n}\right\}$ satisfy $\tau_{n+1}-\sigma_{n} \leq 2 \omega$ for any integer $n$.

Suppose that there exists an $n_{0} \in \mathbb{N}$ such that $\tau_{n_{0}+1}-\sigma_{n_{0}}>2 \omega$. We can choose an $m \in \mathbb{N}$ such that $(m-1) \omega<\sigma_{n_{0}} \leq m \omega$. Hence, we have

$$
\tau_{n_{0}+1}>\sigma_{n_{0}}+2 \omega>(m-1) \omega+2 \omega=(m+1) \omega,
$$

and therefore $[m \omega,(m+1) \omega] \subset\left[\sigma_{n_{0}}, \tau_{n_{0}+1}\right]$. There are two cases to consider: (a) $\bar{p}+p \geq 0$ and (b) $\bar{p}+\underline{p}<0$. In case (a), by Lemma 4 and Remark $2, p(t) \geq \bar{p} / 2>0$ for $\bar{t} \in[a+m \omega, b+\bar{m} \omega] \subset[m \omega,(m+1) \omega]$. Hence, using the second equation in system (11) with (7), (11) and (13), we have

$$
\left|y^{\prime}(t)\right| \geq|p(t)||x(t)|-|q(t)||y(t)|=\frac{1}{2} \bar{p} \sqrt{e^{-2 L} v_{0}-\varepsilon^{2}}-\bar{q} \varepsilon
$$

for $a+m \omega<t<b+m \omega$. It follows from (9) that

$$
\frac{1}{2} \bar{p} \sqrt{e^{-2 L} v_{0}-\varepsilon^{2}}-\bar{q} \varepsilon>\frac{2}{b-a} \varepsilon .
$$

From (11) and (14), we can estimate that

$$
\begin{aligned}
2 \varepsilon & \geq|y(b+m \omega)|+|y(a+m \omega)| \geq\left|\int_{a+m \omega}^{b+m \omega} y^{\prime}(s) d s\right| \\
& =\int_{a+m \omega}^{b+m \omega}\left|y^{\prime}(s)\right| d s \geq(b-a)\left(\frac{1}{2} \bar{p} \sqrt{e^{-2 L} v_{0}-\varepsilon^{2}}-\bar{q} \varepsilon\right) .
\end{aligned}
$$


This contradicts (15). In case (b), by Lemma 4 and Remark $2, p(t) \leq p / 2<0$ for $t \in[a+m \omega, b+m \omega] \subset[m \omega,(m+1) \omega]$. Hence, combining this with (7), (11) and (13), we obtain

$$
\left|y^{\prime}(t)\right| \geq|p(t)||x(t)|-|q(t)||y(t)|=-\frac{1}{2} \underline{p} \sqrt{e^{-2 L} v_{0}-\varepsilon^{2}}-\bar{q} \varepsilon
$$

for $a+m \omega<t<b+m \omega$. It follows from (9) that

$$
-\frac{1}{2} \underline{p} \sqrt{e^{-2 L} v_{0}-\varepsilon^{2}}-\bar{q} \varepsilon>\frac{2}{b-a} \varepsilon .
$$

From (11) and (16), we can estimate that

$$
\begin{aligned}
2 \varepsilon & \geq|y(b+m \omega)|+|y(a+m \omega)| \geq\left|\int_{a+m \omega}^{b+m \omega} y^{\prime}(s) d s\right| \\
& =\int_{a+m \omega}^{b+m \omega}\left|y^{\prime}(s)\right| d s \geq(b-a)\left(-\frac{1}{2} \underline{p} \sqrt{e^{-2 L} v_{0}-\varepsilon^{2}}-\bar{q} \varepsilon\right) .
\end{aligned}
$$

This contradicts (17). Thus, the claim is proved.

Let $I=\bigcup_{n=1}^{\infty}\left[\tau_{n}, \sigma_{n}\right]$. Then, by means of Lemma 2 with (6) and (10), we get

$$
\begin{aligned}
\infty>\int_{t_{0}}^{\infty}\left|v^{\prime}(s)\right| d s & =\frac{1}{2} \int_{t_{0}}^{\infty}|\psi(s)| e^{2 R(s)} y^{2}(s) d s \\
& \geq \frac{1}{2} e^{-2 L} \int_{t_{0}}^{\infty} \psi_{+}(s) y^{2}(s) d s \geq \frac{1}{2} \varepsilon^{2} e^{-2 L} \int_{I} \psi_{+}(s) d s .
\end{aligned}
$$

Hence, it follows from assumption (i) and the Claim that $\liminf _{n \rightarrow \infty}\left(\sigma_{n}-\tau_{n}\right)=0$. Since $\left[t_{n}, s_{n}\right] \subset\left[\tau_{n}, \sigma_{n}\right]$, it follows that

$$
\liminf _{n \rightarrow \infty}\left(s_{n}-t_{n}\right)=0
$$

By (5), (8) and (12), we have

$$
|x(t)|=\sqrt{2 e^{-2 R(t)} v(t)-y^{2}(t)} \leq \sqrt{3 e^{2 L} v_{0}-\frac{\nu^{2}}{4}}
$$

for $t_{n} \leq t \leq s_{n}$. Let $K=\max \{|\bar{p}|,|\underline{p}|\}$. Then, from (77) and (12), we see that

$$
\left|y^{\prime}(t)\right| \leq|p(t)||x(t)|+|q(t)||y(t)|<K \sqrt{3 e^{2 L} v_{0}-\frac{\nu^{2}}{4}}+\frac{3}{4} \bar{q} \nu
$$

for $t_{n} \leq t \leq s_{n}$. Letting $N=K \sqrt{3 e^{2 L} v_{0}-\nu^{2} / 4}+3 \bar{q} \nu / 4$ and integrating this inequality from $t_{n}$ to $s_{n}$, we obtain

$$
\begin{aligned}
\frac{1}{4} \nu & =\left|y\left(s_{n}\right)\right|-\left|y\left(t_{n}\right)\right| \leq\left|y\left(s_{n}\right)-y\left(t_{n}\right)\right| \\
& =\left|\int_{t_{n}}^{s_{n}} y^{\prime}(s) d s\right| \leq \int_{t_{n}}^{s_{n}}\left|y^{\prime}(s)\right| d s \leq N\left(s_{n}-t_{n}\right) .
\end{aligned}
$$

This contradicts (18). We therefore conclude that $\lim \sup _{t \rightarrow \infty}|y(t)|=\nu=0$.

In summary, $y(t)$ tends to zero as $t \rightarrow \infty$. Hence, there exists a $T_{3} \geq T_{2}$ such that

$$
|y(t)|<\varepsilon \quad \text { for } t \geq T_{3} .
$$


Let $l$ be an integer satisfying $l \omega>T_{3}$. Using (19) instead of (11) and following the same process as in the proof of the Claim, we see that if $\bar{p}+\underline{p} \geq 0$, then

$$
\begin{aligned}
2 \varepsilon & \geq|y(b+l \omega)|+|y(a+l \omega)| \geq\left|\int_{a+l \omega}^{b+l \omega} y^{\prime}(s) d s\right| \\
& =\int_{a+l \omega}^{b+l \omega}\left|y^{\prime}(s)\right| d s \geq(b-a)\left(\frac{1}{2} \bar{p} \sqrt{e^{-2 L} v_{0}-\varepsilon^{2}}-\bar{q} \varepsilon\right)>2 \varepsilon,
\end{aligned}
$$

which is a contradiction; if $\bar{p}+\underline{p}<0$, then

$$
\begin{aligned}
2 \varepsilon & \geq|y(b+l \omega)|+|y(a+l \omega)| \geq\left|\int_{a+l \omega}^{b+l \omega} y^{\prime}(s) d s\right| \\
& =\int_{a+l \omega}^{b+l \omega}\left|y^{\prime}(s)\right| d s \geq(b-a)\left(-\frac{1}{2} \underline{p} \sqrt{e^{-2 L} v_{0}-\varepsilon^{2}}-\bar{q} \varepsilon\right)>2 \varepsilon,
\end{aligned}
$$

which is again a contradiction. Thus, the case of $v_{0}>0$ cannot happen.

The proof of Theorem 1 is thus complete.

\section{EXAmples}

We illustrate our main result with simple examples in which $p(t), q(t)$ and $r(t)$ are periodic. It is well-known that if the zero solution of a linear periodic system is attractive, then it is uniformly asymptotically stable (for example, see [5, 18]).

Example 1. Let $\lambda>0$. Consider system (11) with

$$
p(t)=\cos t, \quad q(t)=\frac{\lambda}{2-\sin t} \quad \text { and } \quad r(t)=0 .
$$

Then the zero solution is attractive.

Since $\lambda / 3 \leq q(t) \leq \lambda$ and $R(t) \equiv 0$, it is clear that $q(t)$ and $R(t)$ are bounded for $t \geq 0$. Also, assumptions (i) and (ii) are satisfied. In fact, we have

$$
\psi(t)=2(q(t)-r(t))=\frac{2 \lambda}{2-\sin t},
$$

and therefore

$$
\psi_{+}(t)=\frac{2 \lambda}{2-\sin t} \quad \text { and } \quad \psi_{-}(t)=0
$$

for $t \geq 0$. Hence, $\psi_{+}(t)$ is weakly integrally positive and

$$
\int_{0}^{\infty} \psi_{-}(t) d t=0
$$

Thus, by means of Theorem 1, we conclude that the zero solution is attractive.

Figure 1(a) shows a positive orbit of (1) with (20) and $\lambda=0.1$. The starting point $\mathbf{x}_{0}$ is $(-1,0)$ and the initial time $t_{0}$ is 0 . The positive orbit moves around the origin $\mathbf{0}$ in a clockwise and a counter-clockwise direction alternately, because $p(t)$ changes its sign. The positive orbit approaches the origin $\mathbf{0}$ as it goes up and down.

Example 2. Let $\lambda \geq 1$. Consider system (11) with

$$
p(t)=\cos \lambda t, \quad q(t)=\cos ^{2} t+\sin t \quad \text { and } \quad r(t)=\sin t .
$$

Then the zero solution is attractive. 
It is easy to check that $q(t)$ and $R(t)$ are bounded for $t \geq 0$ and that assumptions (i) and (ii) are satisfied. We omit the details.

In Figure 1(b), we show a positive orbit of (11) with (21) and $\lambda=4$. The positive orbit starts from the point $(-1,0)$ at the initial time 0 . The positive orbit goes to the right and then goes to the left, and it repeats such a movement regularly. Although the positive orbit displays intricate behavior, it approaches the origin $\mathbf{0}$ ultimately.

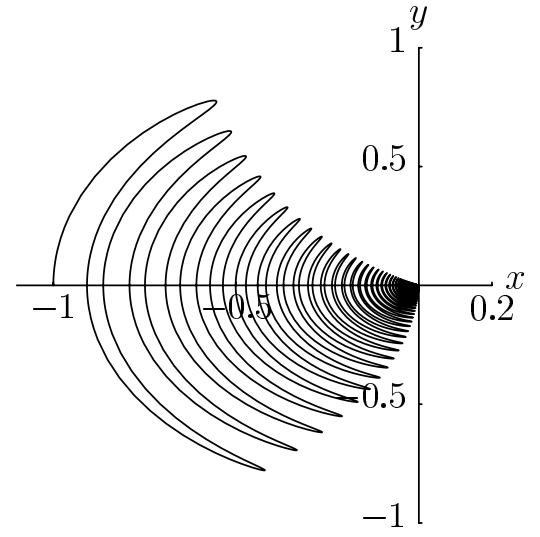

(a)

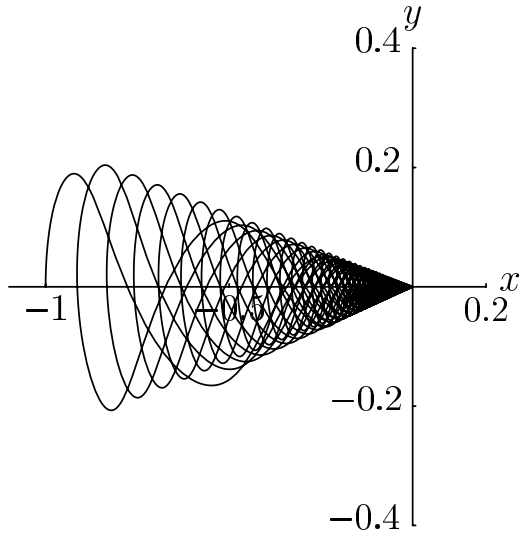

(b)

Figure 1. (a) A positive orbit of (1) with (20); (b) a positive orbit of (1) with (21)

In Examples 1 and 2, all coefficients of (1) are periodic functions with period $2 \pi$. However, we cannot find the monodromy matrix $\Phi(2 \pi)$. It is particularly hard to estimate the absolute value of the trace of $\Phi(2 \pi)$. For this reason, we cannot apply Floquet's theorem to Examples 1 and 2 directly. Theorem 1 has the advantage of being applicable to cases where the monodromy matrix of (11) cannot be found and cases where $q(t)$ or $r(t)$ is not periodic.

\begin{tabular}{c|c|c}
$\lambda$ & $\mu_{1}$ & $\mu_{2}$ \\
\hline 1 & 0.3351718550789 & 0.0793024028529 \\
\hline 0.1 & 0.8888872982404 & 0.7827240687567 \\
\hline 0.01 & 0.9882826823640 & 0.9758079535053 \\
\hline 0.001 & 0.9988220356864 & 0.9975540561378 \\
\hline
\end{tabular}

TABLE 1. Floquet multipliers of (1) with (20)

\begin{tabular}{c|c|c}
$\lambda$ & $\mu_{1}$ & $\mu_{2}$ \\
\hline 1 & 0.5569470757759 & 0.0775907086028 \\
\hline 10 & 0.9845517600942 & 0.0438919719768 \\
\hline 100 & 0.9998429464892 & 0.0432207062297 \\
\hline 1000 & 0.9999986933319 & 0.0432139974342 \\
\hline
\end{tabular}

TABLE 2. Floquet multipliers of (1) with (21) 
Fortunately, in Examples 1 and 2, the Floquet multipliers $\mu_{1}$ and $\mu_{2}$ can be calculated by a numerical scheme. As shown in Tables 1 and $2,\left|\mu_{1}\right|<1$ and $\left|\mu_{2}\right|<1$. Hence, we see that the zero solution of (11) is attractive.

Remark 3. The zero solution of system (11) with (20) is attractive if and only if $\lambda>0$. In fact, if $\lambda \leq 0$, then

$$
\exp \left(-\int_{0}^{\omega}(q(s)+r(s)) d s\right)=\exp \left(-\int_{0}^{\omega} \frac{\lambda}{2-\sin t} d s\right) \geq 1
$$

Hence, as mentioned in Section 1, the zero solution is not attractive in this case.

\section{REFERENCES}

[1] A. Bacciotti and L. Rosier, Liapunov Functions and Stability in Control Theory, 2nd ed., Springer-Verlag, Berlin-Heidelberg-New York, 2005. MR2146587 (2005m:93001)

[2] F. Brauer and J. Nohel, The Qualitative Theory of Ordinary Differential Equations, W. A. Benjamin, New York and Amsterdam, 1969; (revised) Dover, New York, 1989.

[3] J. Cronin, Differential Equations: Introduction and Qualitative Theory, 2nd ed., Monographs and Textbooks in Pure and Applied Mathematics 180, Marcel Dekker, New York-Basel-Hong Kong, 1994. MR1275827 (95b:34001)

[4] A. Halanay, Differential Equations: Stability, Oscillations, Time Lags, Academic Press, New York-London, 1966. MR0216103 (35:6938)

[5] J. K. Hale, Ordinary Differential Equations, Wiley-Interscience, New York-London-Sydney, 1969; (revised) Krieger, Malabar, 1980. MR0419901 (54:7918)

[6] L. Hatvani, On the asymptotic stability by nondecrescent Ljapunov function, Nonlinear Anal., 8 (1984), 67-77. MR732416 (85k:34117)

[7] L. Hatvani, On the asymptotic stability for a two-dimensional linear nonautonomous differential system, Nonlinear Anal., 25 (1995), 991-1002. MR.1350721 (96k:34105)

[8] D. W. Jordan and P. Smith, Nonlinear Ordinary Differential Equations: An Introduction to Dynamical Systems, 3rd ed., Oxford Texts in Applied and Engineering Mathematics 2, Oxford University Press, Oxford, 1999. MR1743361 (2000j:34001)

[9] J. LaSalle and S. Lefschetz, Stability by Liapunov's Direct Method, with Applications, Mathematics in Science and Engineering 4, Academic Press, New York-London, 1961. MR0132876 (24:A2712)

[10] D. R. Merkin, Introduction to the Theory of Stability, Texts in Applied Mathematics 24, Springer-Verlag, New York-Berlin-Heidelberg, 1997. MR.1418401 (98f:34074)

[11] A. N. Michel, L. Hou and D. Liu, Stability of Dynamical Systems: Continuous, Discontinuous, and Discrete Systems, Birkhäuser, Boston-Basel-Berlin, 2008. MR2351563 (2008i:93001)

[12] N. Rouche, P. Habets and M. Laloy, Stability Theory by Liapunov's Direct Method, Applied Mathematical Sciences 22, Springer-Verlag, New York-Heidelberg-Berlin, 1977. MR0450715 (56:9008)

[13] J. Sugie, Convergence of solutions of time-varying linear systems with integrable forcing term, Bull. Austral. Math. Soc., 78 (2008), 445-462. MR2472280

[14] J. Sugie, Influence of anti-diagonals on the asymptotic stability for linear differential systems, Manatsh. Math., 157 (2009), 163-176.

[15] J. Sugie and Y. Ogami, Asymptotic stability for three-dimensional linear differential systems with time-varying coefficients, to appear in Quart. Appl. Math.

[16] F. Verhulst, Nonlinear Differential Equations and Dynamical Systems, Springer-Verlag, New York-Berlin-Heidelberg, 1990. MR.1036522 (91b:34002)

[17] T. Yoshizawa, Note on the boundedness and the ultimate boundedness of solutions of $x^{\prime}=$ $F(t, x)$, Mem. Coll. Sci. Univ. Kyoto Ser. A Math., 29 (1955), 275-291. MR0098217(20:4679) 
[18] T. Yoshizawa, Stability Theory by Liapunov's Second Method, Math. Soc. Japan, Tokyo, 1966. MR0208086 (34:7896)

[19] T. Yoshizawa, Stability Theory and the Existence of Periodic Solutions and Almost Periodic Solutions, Applied Mathematical Sciences 14, Springer-Verlag, New York-Heidelberg-Berlin, 1975. MR0466797 (57:6673)

Department of Mathematics and Computer Science, Shimane University, Matsue 6908504, JAPAN

E-mail address: jsugie@riko.shimane-u.ac.jp

Department of Mathematics and Computer Science, Shimane University, Matsue 6908504, JAPAN 